\title{
Filter Spectral Shaping with Integrated Coupled Sagnac Loop Reflectors in Self-Coupled Nanowires
}

This paper was downloaded from TechRxiv (https://www.techrxiv.org).

\section{LICENSE}

CC BY 4.0

SUBMISSION DATE / POSTED DATE

$18-04-2021$ / 21-04-2021

\section{CITATION}

Moss, David (2021): Filter Spectral Shaping with Integrated Coupled Sagnac Loop Reflectors in Self-Coupled Nanowires. TechRxiv. Preprint. https://doi.org/10.36227/techrxiv.14445633.v1

$\mathrm{DOI}$

10.36227/techrxiv.14445633.v1 


\title{
Filter Spectral Shaping with Integrated Coupled Sagnac Loop Reflectors in Self- Coupled Nanowires
}

\author{
Hamed Arianfard, Jiayang Wu, Member, IEEE, Saulius Juodkazis, Fellow, OSA, \\ and David J. Moss, Fellow, IEEE, Fellow, OSA
}

\begin{abstract}
We propose and theoretically investigate integrated photonic filters based on coupled Sagnac loop reflectors (SLRs) formed by a self-coupled wire waveguide. By tailoring coherent mode interference in the device, three different filter functions are achieved, including Fano-like resonances, wavelength interleaving, and varied resonance mode splitting. For each function, the impact of device structural parameters is analyzed to facilitate optimized performance. Our results theoretically verify the proposed device as a compact multi-functional integrated photonic filter for flexible spectral shaping.
\end{abstract}

Index Terms-Integrated photonic resonators, Sagnac loop reflectors, Fano resonance, interleavers, mode splitting.

\section{INTRODUCTION}

$\mathrm{W}$ ITH a compact footprint, flexible topology, and high scalability, integrated photonic resonators (IPRs) have enabled diverse functional optical devices such as filters, modulators, sensors, switches, and logic gates [1, 2]. As compared with IPRs based on subwavelength gratings [3] and photonic crystal structures [4] that have submicron cavity lengths, IPRs formed by directional-coupled wire waveguides with longer cavity lengths (typically $>10 \mu \mathrm{m}$ ) have smaller free spectral ranges (FSRs) that match with the spectral grids of the state-of-the-art wavelength division multiplexing (WDM) optical communication systems, thus rendering them more widely applicable to these systems. Moreover, the directional-coupled wire waveguides with longer coupling regions and simpler designs also yield a higher tolerance to fabrication imperfections.

Generally, there are two types of basic building blocks for IPRs formed by directional-coupled wire waveguides. The first is a ring resonator, and the second is a Sagnac loop reflector (SLR). In contrast to ring resonators that involve only unidirectional light propagation, the SLRs allow bidirectional light propagation as well as mutual coupling between the light propagating in opposite directions, thus yielding a more versatile coherent mode interference and spectral response. In addition, a standing-wave (SW) resonator formed by cascaded SLRs has a cavity length almost half that of a travelling-wave

This work was supported in part by the Australian Research Council Discovery Projects Programs under Grant DP150102972 and DP190103186, in part by the Swinburne ECR-SUPRA program. (Corresponding authors: Jiayang Wu and David J. Moss).

The authors are with the Optical Sciences Centre, Swinburne University of Technology, Hawthorn, VIC 3122, Australia (e-mail: harianfard@swin.edu.au; jiayangwu@swin.edu.au; sjuodkazis@swin.edu.au; dmoss@swin.edu.au).
(TW) resonator based on a ring resonator with the same FSR, which allows for a more compact device footprint.

In our previous work, we investigated integrated photonic filters based on cascaded SLRs [5, 6] and coupled SLRs [7, 8]. Here, we advance this field by introducing the novel approach of using coupled SLRs formed by a self-coupled wire waveguide. This allows us to achieve versatile spectral responses with a simpler design and a higher fabrication tolerance. We tailor the coherent mode interference to achieve three different filter functions, including Fano-like resonances, wavelength interleaving, and varied resonance mode splitting. The requirements for practical applications are considered in our design. Excellent performance parameters are achieved for each filter function, analysis of the impact of the structural parameters and fabrication tolerance is also provided.

\section{Device Structure}

Fig. 1 illustrates a schematic configuration of the proposed structure, consisting of three SLRs formed by a single selfcoupled wire waveguide. The device structural parameters are defined in Table I. To simplify the discussion, we assume that $L_{\mathrm{SLR} 1}=L_{\mathrm{SLR} 2}=L_{\mathrm{SLR} 3}=L_{\mathrm{SLR}}$ and $L_{1}=L_{2}=L$. The resonator is equivalent to three cascaded SLRs (which is an infiniteimpulse-response (IIR) filter) when $t_{2}=1$ and a SLR with an interferometric coupler [9] (which is a finite-impulse-response (FIR) filter) when $t_{1}=t_{3}=1$. When $t_{i}(i=1-3) \neq 1$, this device is a hybrid filter consisting of both IIR and FIR filter elements, which allows for versatile coherent mode interference and ultimately a diverse range of spectral responses.

We use the scattering matrix method $[5,7]$ to calculate the spectral response of the device.- In our calculation, we assume

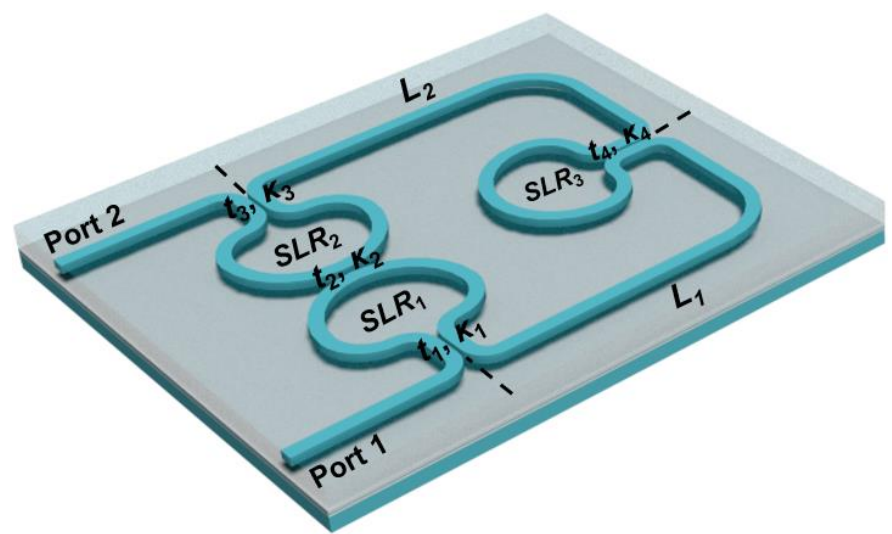

Fig. 1. Schematic configuration of the device configuration consisting of three SLRs $\left(S L R_{1}, S L R_{2}\right.$, and $\left.S L R_{3}\right)$. The definitions of $t_{i}(i=1-4), k_{i}(i=1-4), L_{\mathrm{SLR} i}$ $(i=1-3)$ and $L_{i}(i=1,2)$ are provided in Table I. 
a waveguide group index of $n_{\mathrm{g}}=4.3350$ for transverse electric (TE) mode and a propagation loss of $\alpha=55 \mathrm{~m}^{-1}$ (i.e., 2.4 $\mathrm{dB} / \mathrm{cm}$ ) based on our previously fabricated silicon-on-insulator (SOI) devices [5, 6]. The device is designed based on, but not restricted to, the SOI platform.

TABLE I

DEFINITIONS OF DEVICE STRUCTURAL PARAMETERS

\begin{tabular}{|c|c|c|c|c|}
\hline Waveguides & Length & & $\begin{array}{l}\text { mission } \\
\text { or }^{\text {a }}\end{array}$ & $\begin{array}{l}\text { Phase } \\
\text { shift }^{\text {b }}\end{array}$ \\
\hline $\begin{array}{l}\text { Connecting waveguides } \\
\text { between SLRs }(i=1,2)\end{array}$ & $L_{i}$ & & $a_{i}$ & $\varphi_{i}$ \\
\hline $\begin{array}{c}\text { Sagnac loop in } S L R_{i} \\
(i=1,2,3)\end{array}$ & $L_{\mathrm{SLR} i}$ & & $l_{\mathrm{si}}$ & $\varphi_{\mathrm{si}}$ \\
\hline Directional couplers & \multicolumn{2}{|c|}{$\begin{array}{c}\text { Field transmission } \\
\text { coefficient }^{c}\end{array}$} & \multicolumn{2}{|c|}{$\begin{array}{l}\text { Field cross-coupling } \\
\text { coefficient }^{c}\end{array}$} \\
\hline $\begin{array}{c}\text { Coupler in } S L R s \\
(i=1,3,4)\end{array}$ & \multicolumn{2}{|c|}{ 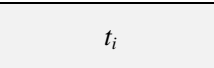 } & \multicolumn{2}{|c|}{ 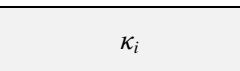 } \\
\hline $\begin{array}{c}\text { Coupler between } S L R_{1} \\
\text { and } S L R_{2}\end{array}$ & \multicolumn{2}{|c|}{$t_{2}$} & \multicolumn{2}{|c|}{$\kappa_{2}$} \\
\hline
\end{tabular}

${ }^{\mathrm{a}} a_{i}=\exp \left(-\alpha L_{i} / 2\right), a_{\mathrm{s} i}=\exp \left(-\alpha L_{\mathrm{SLR} i} / 2\right), \alpha$ is the power propagation loss factor.

b $\varphi_{i}=2 \pi n_{\mathrm{g}} L_{i} / \lambda, \varphi_{\mathrm{si}}=2 \pi n_{\mathrm{g}} L_{\mathrm{SLR} i} / \lambda, n_{\mathrm{g}}$ is the group index and $\lambda$ is the wavelength.

${ }^{\mathrm{c}} t_{\mathrm{si}}{ }^{2}+\kappa_{\mathrm{si}}{ }^{2}=1$ and $t_{\mathrm{b} i}{ }^{2}+\kappa_{\mathrm{b} i}{ }^{2}=1$ for lossless coupling are assumed for all the directional couplers.

\section{FANO-LIKE RESONANCES}

Fano resonances that feature an asymmetric spectral lineshape are fundamental physical phenomena that have underpinned many applications such as optical switching, data storage, sensing, and topological optics [10-12]. In this section, the spectral response of the device in Fig. 1 is tailored to realize optical analogues of Fano resonances with high slope rates (SRs) and low insertion loss (IL). The power transmission and reflection spectra with input from Port 1 is depicted in Fig. 2(a-i). The device structural parameters are $L_{\mathrm{SLR}}=L=100 \mu \mathrm{m}, t_{1}=t_{3}=0.82, t_{2}=0.92$, and $t_{4}=1$. Clearly, the output from Port 2 shows periodical Fano-like resonances with an asymmetric resonant lineshape in each period. The high uniformity of the filter shape across multiple periods, or channels, is highly desirable for WDM systems. A zoom-in view of Fig. 2(a-i) is shown in Fig. 2(a-ii), together with another curve showing the corresponding result for another device with the same structural parameters except for a different $t_{2}=1$. As can be seen, when $t_{2}=1$, there is no Fano resonance, distinguishing between the device in Fig. 1 and the three cascaded SLRs in Ref. [5]. The Fano resonances in Fig. 2(a-ii) show a high extinction ratio (ER) of $30.2 \mathrm{~dB}$ and a high SR (defined as the ratio of the ER to the wavelength difference

TABLE II

PERFORMANCE COMPARISON OF FANO-LIKE RESONANCES GENERATED BY DIFFERENT SLR-BASED DEVICES

\begin{tabular}{c|c|c|c|c|c}
\hline \hline Device structure & $\begin{array}{c}\text { IL } \\
(\mathbf{d B})\end{array}$ & $\begin{array}{c}\text { ER } \\
(\mathbf{d B})\end{array}$ & $\begin{array}{c}\text { SR } \\
(\mathbf{d B} / \mathbf{n m})\end{array}$ & $\begin{array}{c}\text { FSR } \\
(\mathbf{G H z})\end{array}$ & Ref. \\
\hline $\begin{array}{c}\text { Two parallel } \\
\text { WC-SLRs }\end{array}$ & 6.3 & 13.9 & 389 & 692.02 & {$[7]$} \\
\hline $\begin{array}{c}\text { Three zig-zag } \\
\text { WC-SLRs }\end{array}$ & 3.7 & 63.4 & 721.28 & 230.68 & {$[8]$} \\
\hline Device in Fig. 1 & 1.1 & 30.2 & 747.64 & 173 & $\begin{array}{c}\text { This } \\
\text { work }\end{array}$ \\
\hline \hline
\end{tabular}

${ }^{a}$ WC-SLRs: waveguide coupled SLRs.

${ }^{\mathrm{b}}$ For comparison, the length of the SLRs $\left(L_{\mathrm{SLR} i}, i=1-3\right)$ and the connecting waveguide $\left(L_{i}, i=1-4\right)$ is slightly changed from $115 \mu \mathrm{m}$ in [8] to $100 \mu \mathrm{m}$.
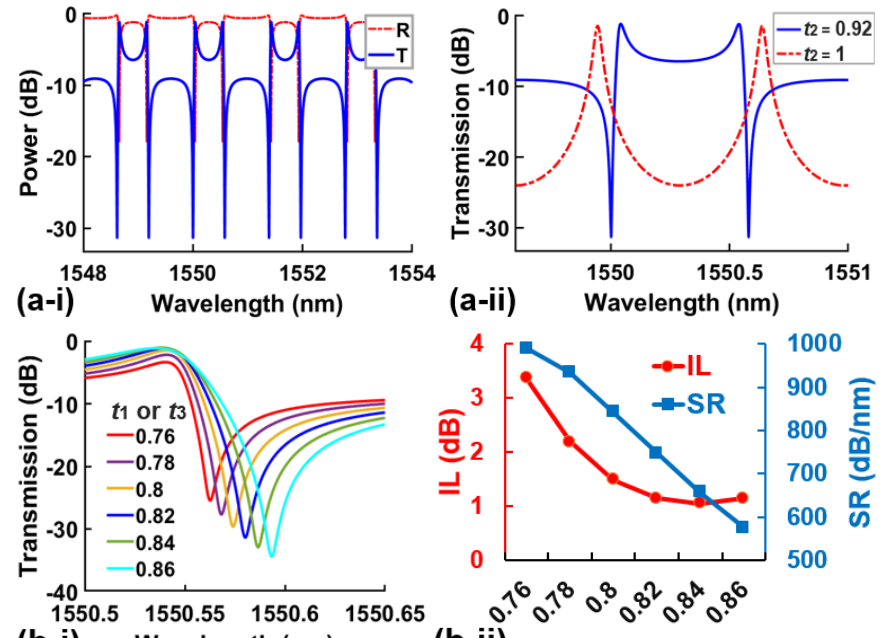

(b-i) Wavelength (nm)
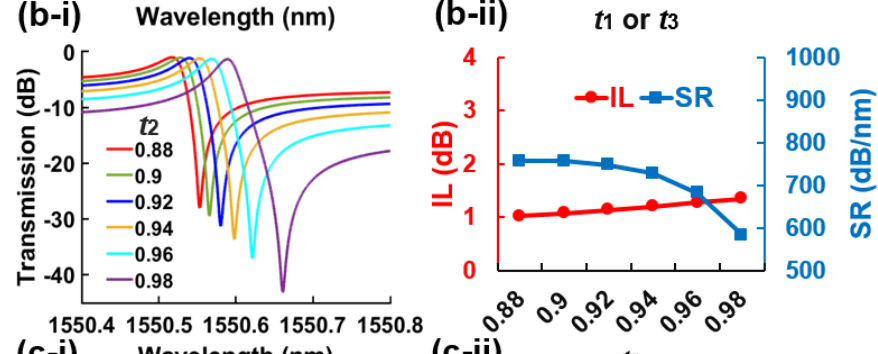

(c-i) Wavelength (nm)

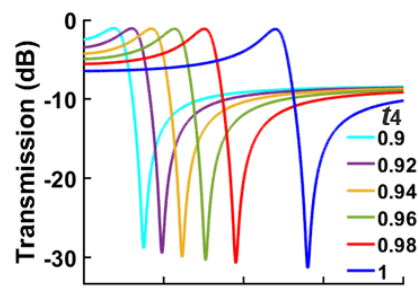

1550.31550 .41550 .51550 .61550 .7

(d-i) Wavelength $(\mathrm{nm})$

(c-ii) $\quad$ t2

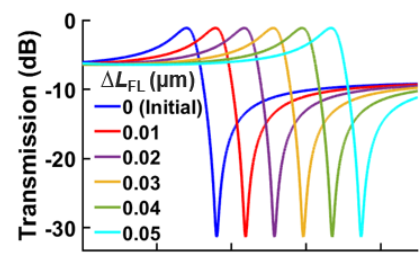

1550.41550 .51550 .61550 .71550 .8 (e-i) Wavelength (nm)

Fig. 2. (a-i) Power transmission and reflection spectra with input from Port 1 when $L_{\mathrm{SLR}}=L=100 \mu \mathrm{m}, t_{1}=t_{3}=0.82, t_{2}=0.92$, and $t_{4}=1$. T: Transmission spectrum at Port 2. R: reflection spectrum at Port 1. (a-ii) Power transmission spectra at Port 2 for $t_{2}=0.92$ and $t_{2}=1$. In (a-ii), the structural parameters are kept the same as those in (a-i) except for $t_{2}$. (b)-(e) (i) Power transmission spectra and (ii) the corresponding IL and SR for different $t_{i}(i=1-4)$ and variation of feedback loop length $\Delta L_{\mathrm{FL}}$, respectively. In (b)-(e), the structural parameters are kept the same as those in (a-i) except for the varied parameters.

between the resonance peak and notch) of $747.64 \mathrm{~dB} / \mathrm{nm}$. Table II compares the performance of the Fano-like resonances generated by the coupled SLRs in our previous work [7, 8] and the device in Fig. 1. As compared with previous devices, the device reported here has a much lower insertion loss of $1.1 \mathrm{~dB}$, along with a slightly improved SR. We note that a low IL of $1.1 \mathrm{~dB}$ is outstanding among the reported Fano-resonance devices on the SOI platform [13, 14], 
which renders the device here more attractive for practical applications in optical communication systems.

In Figs. 2(b)-(e), we investigate the impact of the device structural parameters including $t_{i}(i=1-4)$ and length variations of the feedback loop $\left(\Delta L_{\mathrm{FL}}, L_{\mathrm{FL}}=2 L+L_{\mathrm{SLR}}\right)$, respectively. In each figure, we changed only one structural parameter, keeping the others the same as those in Fig. 2(a-i). Figs. 2(b-i) and (b-ii) compares the power transmission spectra and corresponding IL and SR for various $t_{1}$ or $t_{3}$, respectively. The SR decreases with $t_{i}(i=1,3)$, while the IL first decreases with $t_{i}(i=1,3)$ and then remains almost unchanged. The spectral response and corresponding IL and SR for different $t_{2}$ are shown in Figs. 2(c-i) and (c-ii), respectively. The SR decreases with $t_{2}$, while the IL shows an opposite trend, reflecting that both of the two parameters can be improved by enhancing the coupling strength between $\mathrm{SLR}_{1}$ and $\mathrm{SLR}_{2}$. As shown in Fig. 2(d), both IL and SR remain almost unchanged with varied $t_{4}$. In Figs. 2(e-i) and (e-ii), we compare the corresponding results for various $\Delta L_{\mathrm{FL}}$. As $\Delta L_{\mathrm{FL}}$ increases, the filter shape remains unchanged while the resonance redshifts, indicating that the resonance wavelengths can be tuned by introducing thermo-optic micro-heaters [14] or carrierinjection electrodes [15] along the feedback loop to tune the phase shift.

\section{WAVELENGTH DE-INTERLEAVING}

Optical interleavers and de-interleavers are core elements for signal multiplexing and demultiplexing in WDM optical communication systems $[16,17]$. In this section, we engineer the spectral response of the device in Fig. 1 to achieve wavelength de-interleaving function. Fig. 3(a) shows the power transmission and reflection spectra with input from Port 1. The device structural parameters are $L_{\mathrm{SLR}}=L=100 \mu \mathrm{m}, t_{1}=$ $0.992, t_{2}=t_{3}=0.95$, and $t_{4}=1$. The input signal is separated into two spectrally interleaved signals, with one group transmitting to Port 2 and the other reflecting back at Port 1. The IL, ER, and 3-dB bandwidth for the passband at Port 2 are $0.36 \mathrm{~dB}, 12.7 \mathrm{~dB}$, and $83.65 \mathrm{GHz}$, respectively. The IL, ER, and 3-dB bandwidth for the passband at Port 1 are $0.33 \mathrm{~dB}, 12$ $\mathrm{dB}$, and $91.9 \mathrm{GHz}$, respectively.

We also investigate the impact of varied $t_{i}(i=1-4), \Delta L_{\mathrm{FL}}$, and $\Delta L_{\mathrm{SLR} i}(i=1,2)$ in Figs. 3(b)-(h), respectively. For simplification, we only show the spectral response at Port 2 . In Fig. 3(b), as $t_{1}$ increases, the ER of the passband decreases while the top flatness improves, reflecting the trade-off between them. In Figs. 3(c)-(e), the bandwidth of the passband increases with $t_{2}, t_{3}, t_{4}$, respectively, while the ER shows an opposite trend. In Figs. 3(f)-(h), as $\Delta L_{\mathrm{FL}}$ or $\Delta L_{\mathrm{SLR} i}(i$ $=1,2$ ) increases, the filter shape remains unchanged while the resonance redshifts, indicating the feasibility of achieving tunable de-interleavers with this approach. Since the resonant cavity of the device is formed by a single self-coupled wire waveguide, random length fabrication errors in each part will not induce any asymmetry in the filter shape. This yields a higher fabrication tolerance as compared with the coupled SLRs in Refs. [7, 8], which is particularly attractive for optical interleavers that require a flat-top symmetric filter shape. Note

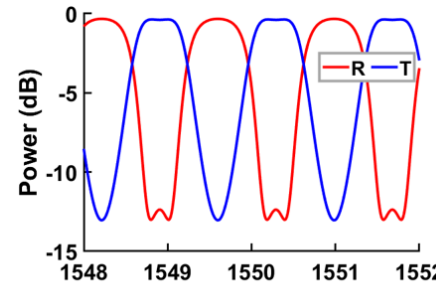

(a)

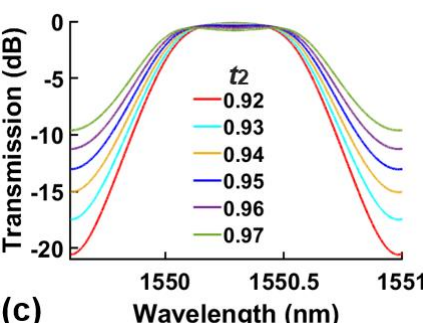

(c)

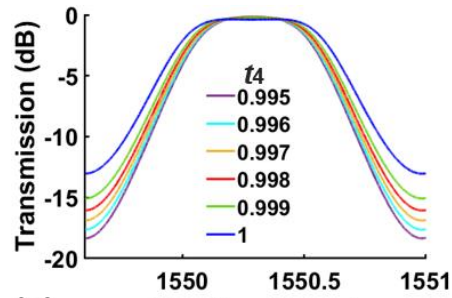

(e) Wavelength $(\mathrm{nm})$

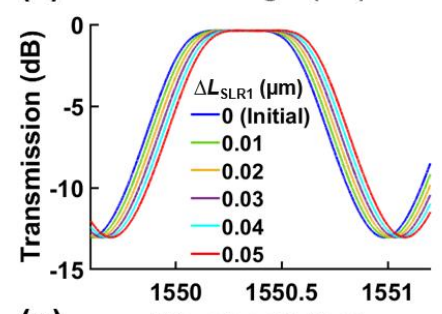

(g)

Wavelength $(\mathrm{nm})$

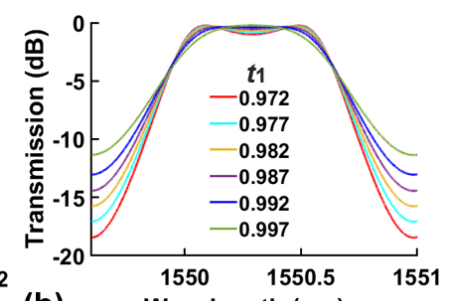

(b) Wavelength (nm)

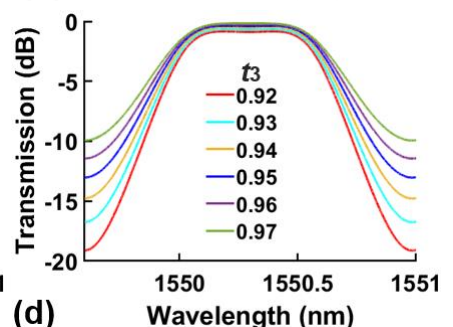

(d) Wavelength $(\mathrm{nm})$

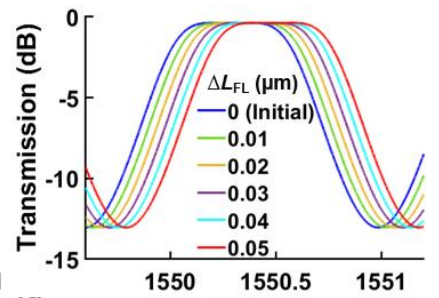

(f) Wavelength $(\mathrm{nm})$

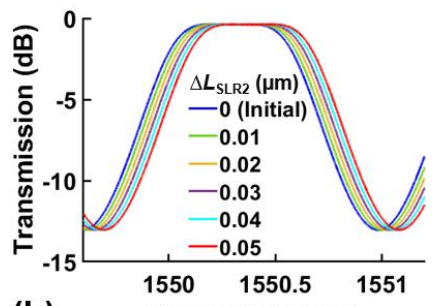

(h) Wavelength $(\mathrm{nm})$

Fig. 3. (a) Power transmission and reflection spectra with input from Port 1 when $L_{\mathrm{SLR}}=L=100 \mu \mathrm{m}, t_{1}=0.992, t_{2}=t_{3}=0.95$, and $t_{4}=1$. T: Transmission spectrum at Port 2. R: reflection spectrum at Port 1. (b)-(h) Power transmission spectra for different $t_{i}(i=1-4), \Delta L_{\mathrm{FL}}$, and $\Delta L_{\mathrm{SLR} i}(i=1,2)$, respectively. In (b)-(h), the structural parameters are kept the same as those in (a) except for the varied parameters.

that the de-interleaving function is designed for the telecom $\mathrm{C}$ band. According to our previous fabricated devices [17], the slight variation in $t_{i}(i=1-4)$ arising from the dispersion of silicon would not significantly deteriorate the periodical response across this wavelength range.

\section{VARIED RESONANCE MODE SPLITTING}

Resonance mode splitting in IPRs induced by coherent mode interference can yield a range of highly useful spectral responses, including electromagnetically induced transparency (EIT), electromagnetically induced absorption (EIA), and Autler-Towns splitting, which have been used for applications such as optical buffering, signal multicasting, analog signal computing, and sensing $[9,18,19]$. In this section, we tailor the spectral response of the device in Fig. 1 to achieve varied resonance mode splitting with diverse spectral response.

Figs. 4(a) and (b) shows the power transmission and reflection spectra for various $t_{4}$, respectively. The input is from Port 1 and the structural parameters are $L_{\mathrm{SLR}}=L=100 \mu \mathrm{m}, t_{1}=$ $t_{3}=0.825$, and $t_{2}=0.99$. As can be seen, by increasing the 


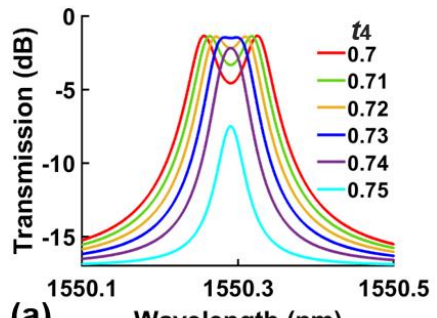

(a) Wavelength $(\mathrm{nm})$
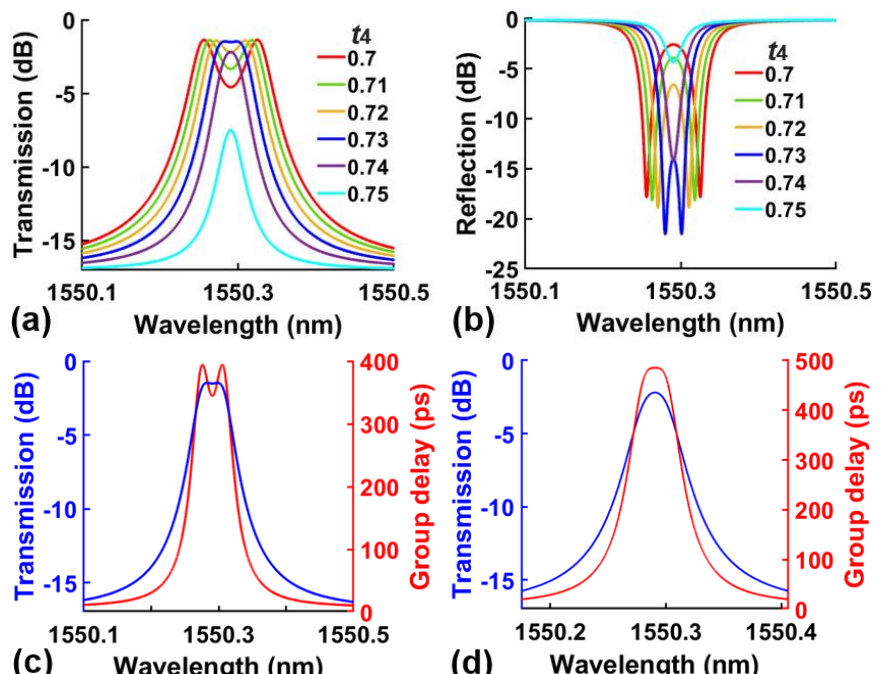

(c) (b) Wavelength $(\mathrm{nm})$

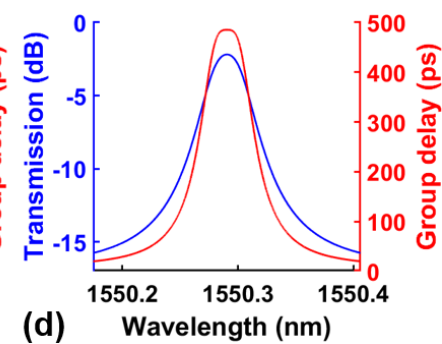

(d)
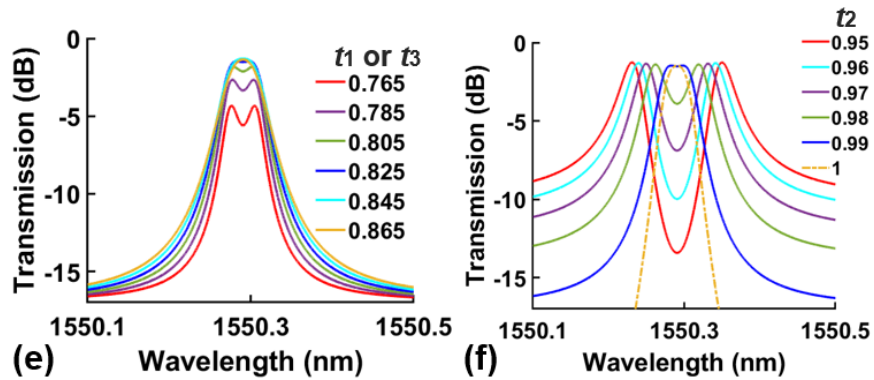

Fig. 4. (a) Power transmission and (b) reflection spectra versus $t_{4}$ when $t_{1}=t_{3}$ $=0.825, t_{2}=0.99$, and $L_{\mathrm{SLR}}=L=100 \mu \mathrm{m}$, respectively. The input is from Port 1. (c)-(d) Power transmission spectrum and corresponding group delay response of (c) a Butterworth filter and (d) a Bessel filter when $t_{4}=0.73$ and $t_{4}=0.74$ in (a), respectively. (e)-(f) Power transmission spectra of the Butterworth filter versus $t_{1}$ or $t_{3}$, and $t_{2}$, respectively. In (e)-(f), the structural parameters are kept the same as those in (c) except for the varied parameters.

coupling strength of the directional coupler in $\mathrm{SLR}_{3}$ (i.e., reducing $t_{4}$ ), the single resonance is gradually split into two resonances with an increased spectral range between them. This is a typical phenomenon for resonance mode splitting similar to those in Ref. [9]. The energy coupling between the light propagating in opposite directions can be changed by varying the reflectivity of $\mathrm{SLR}_{3}$, thus resulting in different mode splitting degrees. Figs. 4(c) and (d) show the power transmission spectrum and group delay response of a Butterworth filter and a Bessel filter formed by resonance mode splitting, respectively. The structural parameters are the same as those in Fig. 4(a) except for a different $t_{4}$. As shown in Fig. 4(e), the Butterworth filter shape gradually transits to a Chebyshev Type I filter shape by further decreasing $t_{1}$ or $t_{3}$. In Fig. 4(f), we compare the spectral response for various $t_{2}$. It can be seen that more significant resonance mode splitting can be obtained by enhancing the coupling strength between SLR $_{1}$ and $\mathrm{SLR}_{2}$ (i.e., reducing the $t_{2}$ ). In particular, when $t_{2}=1$ (which corresponds to three cascaded SLRs), the resonance is still not split, this indicates that the device reported here shows a significantly enhanced resonance mode splitting as compared with the three cascaded SLRs in Ref. [5].

Finally, this work could have applications to nonlinear devices [20-30] as well as to microwave photonic chips [3163] and integrated quantum optics [64- ] where advanced optical filter shapes are extremely useful.

\section{CONCLUSIONS}

We theoretically investigate integrated photonic filters based on coupled SLRs formed by a self-coupled wire waveguide. Three different filter functions have been realized, including Fano-like resonances, wavelength interleaving, and varied resonance mode splitting. The compact footprint, versatile spectral responses, and high fabrication tolerance make this approach highly promising for flexible spectral shaping in a diverse range of applications.

Competing interests: The authors declare no competing interests.

\section{REFERENCES}

[1] W. Bogaerts et al., "Silicon microring resonators," Laser Photon. Rev., vol. 6, no. 1, pp. 47-73, Jan. 2012.

[2] Z. Yao et al., "Integrated silicon photonic microresonators: emerging technologies," IEEE J. Sel. Top. Quantum Electron., vol. 24, no. 6, pp. 124, Nov.-Dec. 2018.

[3] P. Cheben et al., "Subwavelength integrated photonics," Nature, vol. 560, no. 7720, pp. 565-572, Aug. 2018.

[4] T. Gu et al., "Regenerative oscillation and four-wave mixing in graphene optoelectronics," Nat. Photon., vol. 6, no. 8, pp. 554-559, Aug. 2012.

[5] J. Wu et al. "Advanced photonic filters based on cascaded Sagnac loop reflector resonators in silicon-on-insulator nanowires," APL Photon., vol. 3, no. 4, Apr. 2018, Art. no. 046102.

[6] J. Wu et al., "Micro-ring resonator quality factor enhancement via an integrated Fabry-Perot cavity," APL Photon., vol. 2, no. 5, Apr. 2017, Art. no. 056103.

[7] H. Arianfard et al., "Advanced multi-functional integrated photonic filters based on coupled Sagnac loop reflectors," J. Lightw. Technol., vol. 39, no. 5, pp. 1400-1408, Mar. 2021.

[8] H. Arianfard et al., "Three Waveguide Coupled Sagnac Loop Reflectors for Advanced Spectral Engineering," J. Lightwave Technol., Mar. 2021. Early Access: doi: 10.1109/JLT.2021.3066256.

[9] J. Wu et al., "On-chip tunable second-order differential-equation solver based on a silicon photonic mode-split microresonator," J. Lightw. Technol., vol. 33, no. 17, pp. 3542-3549, Sep. 2015.

[10] M. F. Limonov et al., "Fano resonances in photonics," Nat. Photon.., vol. 11, pp. 543-554, Sep. 2017.

[11] E. Kamenetskii, A. Sadreev, and A. Miroshnichenko, "Fano resonances in optics and microwaves: physics and applications," Springer International Publishing, 2018.

[12] E. Miroshnichenko, S. Flach, and Y. S. Kivshar, "Fano resonances in nanoscale structures," Rev. Mod. Phys., vol. 82, no. 3, pp. 2257-2298, Aug. 2010.

[13] A. Li and W. Bogaerts, "An actively controlled silicon ring resonator with a fully tunable Fano resonance," APL Photonics, vol. 2, no. 9, Aug. 2017, Art. no. 096101.

[14] T. Zhao et al., "Independently tunable double Fano resonances based on waveguide-coupled cavities," Opt. Lett., vol. 44, no. 12, pp. 3154-3157, Jun. 2019.

[15] M. C. Souza et al., "Spectral engineering with coupled microcavities: active control of resonant mode-splitting," Opt. Lett., vol. 40, no. 14, pp. 3332-5, Jul 2015.

[16] S. Cao et al., "Interleaver technology: comparisons and applications requirements," J. Lightwave Technol., vol. 22, no. 1, pp. 281-289, Jan. 2004.

[17] S. Lai et al., "Compact silicon photonic interleaver based on a selfcoupled optical waveguide," Appl. Opt., vol. 55, no. 27, pp. 7550-5, Sep. 2016.

[18] L. A. M. Barea et al., "Silicon technology compatible photonic molecules for compact optical signal processing," Appl. Phys. Lett., vol. 103, no. 20, 2013, Art. no. 201102.

[19] L. A. M. Barea et al., "Spectral engineering with CMOS compatible SOI photonic molecules," IEEE Photonics J., vol. 5, no. 6, pp. 22027172202717, Dec. 2013. 
[20] Y. Qu, J. Wu, Y. Yang, Y. Zhang, Y. Liang, H. El Dirani, R. Crochemore, P. Demongodinc, C. Sciancalepore, C. Grillet, C. Monat, B. Jia, D. J. Moss, "Enhanced nonlinear four-wave mixing in silicon nitride waveguides integrated with 2D layered graphene oxide films", Adv. Opt. Mater., vol. 8, no. 20, pp. 2001048 Oct. 2020. DOI: 10.1002/adom.202001048.

[21] Y. Yang, H. Lin, B. Zhang, Y. Zhang, X. Zheng, A. Yu, M. Hong, and B. Jia, "Graphene-based multilayered metamaterials with phototunable architecture for on-chip photonic devices," ACS Photonics, vol. 6, no. 4, pp. 1033-1040, Feb. 2019

[22] J. Wu, Y. Yang, Y. Qu, X. Xu, Y. Liang, S. T. Chu, B. E. Little, R. Morandotti, B. Jia, and D. J. Moss, "Graphene oxide waveguide and micro-ring resonator polarizers," Laser Photonics Rev., vol. 13, no. 9, 1900056, Sep. 2019.

[23] J. Wu, L. Jia, Y. Zhang, Y. Qu, B. Jia, and D. J. Moss, "Graphene oxide for integrated photonics and flat optics," Adv. Mater., vol. 33, no. 3, pp. 200415, Jan. 2021.

[24] J. Wu, Y. Yang, Y. Qu, L. Jia, Y. Zhang, X. Xu, S. T. Chu, B. E. Little, R. Morandotti, B. Jia, and D. J. Moss, "2D layered graphene oxide films integrated with micro-ring resonators for enhanced nonlinear optics," Small, e1906563, Mar. 11 (2020).

[25] Y. Zhang, J. Wu, Y. Yang, Y. Qu, L. Jia, T. Moein, B. Jia, and D. J. Moss, "Enhanced kerr nonlinearity and nonlinear figure of merit in silicon nanowires integrated with 2D graphene oxide films," ACS Applied Materials \& Interfaces, vol. 12, no. 29, pp. 33094-33103, Jul. 2020.

[26] Y. Yang, J. Wu, X. Xu, Y. Liang, S. T. Chu, B. E. Little, R. Morandotti, B. Jia, and D. J. Moss, "Invited Article: Enhanced four-wave mixing in waveguides integrated with graphene oxide," APL Photonics, vol. 3, no. 12, pp. 120803 , Oct. 2018

[27] Y. Zhang, J. Wu, Y. Qu, L. Jia, B. Jia, and D. J. Moss, "Optimizing the Kerr nonlinear optical performance of silicon waveguides integrated with 2D graphene oxide films", Journal of Lightwave Technology, Vol. 39, Early Access (2021). DOI: 10.1109/JLT.2021.3069733

[28] Y. Qu, J. Wu, Y. Zhang, Y. Liang, B. Jia, and D. J. Moss, "Analysis of four-wave mixing in silicon nitride waveguides integrated with $2 \mathrm{D}$ layered graphene oxide films", Journal of Lightwave Technology, vol. 39, Early Access (2021). DOI: 10.1109/JLT.2021.3059721.

[29] L. Jia, J. Wu, Y. Yang, Y. Du, B. Jia, D. J. Moss, "Large Third-Order Optical Kerr Nonlinearity in Nanometer-Thick PdSe2 2D Dichalcogenide Films: Implications for Nonlinear Photonic Devices", ACS Applied Nano Materials, Vol. 3, (7) pp.6876-6883 June 29 (2020). DOI:10.1021/acsanm.0c01239.

[30] L. Jia, D. Cui, J. Wu, H. Feng, T. Yang, Y. Yang, Y. Du, W. Hao, B. Jia, D. J. Moss, "BiOBr nanoflakes with strong nonlinear optical properties towards hybrid integrated photonic devices", Applied Physics Letters (APL) Photonics, vol. 4, 090802 (2019). DOI: 10.1063/1.5116621

[31] X. Xu, et al., "Broadband RF channelizer based on an integrated optical frequency Kerr comb source," Journal of Lightwave Technology, vol. 36, no. 19 , pp. 4519-4526 (2018).

[32] X. Xu, et al., "Continuously tunable orthogonally polarized RF optical single sideband generator based on micro-ring resonators," Journal of Optics, vol. 20, no. 11, 115701 (2018).

[33] X. Xu, et al., "Orthogonally polarized RF optical single sideband generation and dual-channel equalization based on an integrated microring resonator," Journal of Lightwave Technology, vol. 36, no. 20, pp. 4808-4818 (2018).

[34] X. Xu, et al., "Photonic microwave true time delays for phased array antennas using a $49 \mathrm{GHz}$ FSR integrated optical micro-comb source," Photonics Res, vol. 6, no. 5, pp. B30-B36 (2018).

[35] X. Xu, et al., "Advanced adaptive photonic RF filters with 80 taps based on an integrated optical micro-comb source," Journal of Lightwave Technology, vol. 37, no. 4, pp. 1288-1295 (2019).

[36] X. Xu et al., Broadband microwave frequency conversion based on an integrated optical micro-comb source", Journal of Lightwave Technology, vol. 38 no. 2, pp. 332-338 (2020).

[37] M. Tan et al., "Photonic RF and microwave filters based on $49 \mathrm{GHz}$ and $200 \mathrm{GHz}$ Kerr microcombs", Optics Comm. vol. 465, Article: 125563, Feb. $22(2020)$.

[38] X. Xu et al., "Broadband photonic RF channelizer with 90 channels based on a soliton crystal microcomb", Journal of Lightwave Technology, Vol. 38, no. 18, pp.5116 - 5121 (2020). doi: 10.1109/JLT.2020.2997699.

[39] X. Xu et al., "Photonic RF and microwave integrator with soliton crystal microcombs", IEEE Transactions on Circuits and Systems II: Express $\begin{array}{llllll}\text { Briefs, } & \text { Vol. } & 67 & \text { (12) } & 3582-3586 & \text { (2020). }\end{array}$ DOI:10.1109/TCSII.2020.2995682.
[40] X. Xu et al., "Photonic RF phase-encoded signal generation with a microcomb source", Journal of Lightwave Technology, vol. 38, no. 7, pp. $1722-1727(2020)$

[41] X. Xu et al., "High performance RF filters via bandwidth scaling with Kerr micro-combs," APL Photonics, vol. 4, no. 2, pp. 026102 (2019).

[42] M. Tan et al., "Microwave and RF photonic fractional Hilbert transformer based on a $50 \mathrm{GHz}$ Kerr micro-comb", Journal of Lightwave Technology, vol. 37, no. 24, pp. 6097 - 6104 (2019).

[43] M. Tan et al., "RF and microwave fractional differentiator based on photonics", IEEE Transactions on Circuits and Systems: Express Briefs, Vol. 67, No. 11, pp. $2767 \quad$ - $2771 \quad$ (2020). DOI:10.1109/TCSII.2020.2965158.

[44] M. Tan et al., "Photonic RF arbitrary waveform generator based on a soliton crystal micro-comb source", Journal of Lightwave Technology, Vol. 38, No. 22, pp. 6221-6226, Oct 22 (2020). DOI: 10.1109/JLT.2020.3009655.

[45] M. Tan, X. Xu, J. Wu, R. Morandotti, A. Mitchell, and D. J. Moss, "RF and microwave high bandwidth signal processing based on Kerr Microcombs", Advances in Physics X, VOL. 6, NO. 1, 1838946 (2020). DOI:10.1080/23746149.2020.1838946.

[46] Mengxi Tan, Xingyuan Xu, Jiayang Wu, Thach G. Nguyen, Sai T. Chu, Brent E. Little, Roberto Morandotti, Arnan Mitchell, and David J. Moss, "Photonic Radio Frequency Channelizers based on Kerr Micro-combs and Integrated Micro-ring Resonators", JOSarXiv.202010.0002.

[47] M. Tan, X. Xu, D.J. Moss "Tunable Broadband RF Photonic Fractional Hilbert Transformer Based on a Soliton Crystal Microcomb", Preprints, DOI: 10.20944/preprints202104.0162.v1

[48] M. Tan, X. Xu, J. Wu, T. G. Nguyen, S. T. Chu, B. E. Little, R. Morandotti, A. Mitchell, and D. J. Moss, "Orthogonally polarized Photonic Radio Frequency single sideband generation with integrated micro-ring resonators", Journal of Semiconductors $\underline{42}$ (4), 041305 (2021). DOI: $10.1088 / 1674-4926 / 42 / 4 / 041305$.

[49] M. Tan, X. Xu, J. Wu, T. G. Nguyen, S. T. Chu, B. E. Little, R. Morandotti, A. Mitchell, and D. J. Moss, "Photonic Radio Frequency Channelizers based on Kerr Optical Micro-combs", Journal of Semiconductors $\mathbf{4 2}$ (4), $041302 \quad$ (2021). (ISSN 1674-4926). DOI: $10.1088 / 1674-4926 / 42 / 4 / 041302$.

[50] H.Bao, L.Olivieri, M.Rowley, S.T. Chu, B.E. Little, R.Morandotti, D.J. Moss, J.S.T. Gongora, M.Peccianti and A.Pasquazi, "Laser Cavity Solitons and Turing Patterns in Microresonator Filtered Lasers: properties and perspectives", Paper No. LA203-5, Paper No. 11672-5, SPIE LASE, SPIE Photonics West, San Francisco CA March 6-11 (2021). DOI: $10.1117 / 12.2576645$

[51] M. Tan, X. Xu, J. Wu, A. Boes, T. G. Nguyen, S. T. Chu, B. E. Little, R. Morandotti, A. Mitchell, and D. J. Moss, "Advanced microwave signal generation and processing with soliton crystal microcombs", or "Photonic convolutional accelerator and neural network in the Tera-OPs regime based on Kerr microcombs", Paper No. 11689-38, PW21O-OE201-67, Integrated Optics: Devices, Materials, and Technologies XXV, SPIE Photonics West, San Francisco CA March 6-11 (2021). DOI: $10.1117 / 12.2584017$

[52] M. Tan, B. Corcoran, X. Xu, A. Boes, J. Wu, T. Nguyen, S. T. Chu, B. E. Little, R. Morandotti, A. Mitchell, and D. J. Moss, "Optical data transmission at 40 Terabits/s with a Kerr soliton crystal microcomb", Paper No.11713-8, PW21O-OE803-23, Next-Generation Optical Communication: Components, Sub-Systems, and Systems X, SPIE Photonics West, San Francisco CA March 6-11 (2021). DOI: $10.1117 / 12.2584014$

[53] M. Tan, X. Xu, J. Wu, A. Boes, T. G. Nguyen, S. T. Chu, B. E. Little, R Morandotti, A. Mitchell, and D. J. Moss, "RF and microwave photonic, fractional differentiation, integration, and Hilbert transforms based on Kerr micro-combs", Paper No. 11713-16, PW21O-OE803-24, NextGeneration Optical Communication: Components, Sub-Systems, and Systems X, SPIE Photonics West, San Francisco CA March 6-11 (2021). DOI:10.1117/12.2584018

[54] M. Tan, X. Xu, J. Wu, A. Boes, T. G. Nguyen, S. T. Chu, B. E. Little, R. Morandotti, A. Mitchell, and D. J. Moss, "Broadband photonic RF channelizer with 90 channels based on a soliton crystal microcomb", or "Photonic microwave and RF channelizers based on Kerr micro-combs", Paper No. 11685-22, PW21O-OE106-49, Terahertz, RF, Millimeter, and Submillimeter-Wave Technology and Applications XIV, SPIE Photonics West, San Francisco CA March 6-11 (2021). DOI: $10.1117 / 12.2584015$ 
[55] X. Xu, M. Tan, J. Wu, S. T. Chu, B. E. Little, R. Morandotti, A. Mitchell, B. Corcoran, D. Hicks, and D. J. Moss, "Photonic perceptron based on a Kerr microcomb for scalable high speed optical neural networks", IEEE Topical Meeting on Microwave Photonics (MPW), pp. 220224,.Matsue, Japan, November 24-26, 2020. Electronic ISBN:978-488552-331-1. DOI: 10.23919/MWP48676.2020.9314409

[56] M. Tan, B.Corcoran, X. Xu, A. Boes, J. Wu, T. Nguyen, S.T. Chu, B. E. Little, R. Morandotti, A. Mitchell, and D. J. Moss, "Ultra-high bandwidth optical data transmission with a microcomb", IEEE Topical Meeting on Microwave Photonics (MPW), pp. 78-82.Virtual Conf., Matsue, Japan, November 24-26, 2020. Electronic ISBN:978-4-88552-331-1. DOI: $10.23919 / \mathrm{MWP} 48676.2020 .9314476$

[57] T. G. Nguyen et al., "Integrated frequency comb source-based Hilbert transformer for wideband microwave photonic phase analysis," Opt. Express, vol. 23, no. 17, pp. 22087-22097 (2015)

[58] X. Xu, J. Wu, M. Shoeiby, T. G. Nguyen, S. T. Chu, B. E. Little, R. Morandotti, A. Mitchell, and D. J. Moss, "Reconfigurable broadband microwave photonic intensity differentiator based on an integrated optical frequency comb source," APL Photonics, vol. 2, no. 9, 096104 (2017).

[59] X. Xu, M. Tan, J. Wu, R. Morandotti, A. Mitchell, and D. J. Moss, "Microcomb-based photonic RF signal processing", IEEE Photonics Technology Letters, vol. 31 no. 23 1854-1857 (2019).

[60] X. Xu, et al., "Advanced RF and microwave functions based on an integrated optical frequency comb source," Opt. Express, vol. 26, no. 3, pp. 2569-2583 (2018).

[61] B. Corcoran, M. Tan, X. Xu, A. Boes, J. Wu, T. G. Nguyen, S. T. Chu, B. E. Little, R. Morandotti, A. Mitchell and D. J. Moss, "Ultra-dense optical data transmission over standard fibre with a single chip source," Nat. Comm., vol. 11, no. 1, pp. 2568, May 2020.

[62] X. Xu, et al., "Photonic perceptron based on a Kerr microcomb for scalable high speed optical neural networks", Laser and Photonics Reviews, vol. 14, no. 10, pp. 2000070, 2020.

[63] X. Xu, et al., "11 TOPS photonic convolutional accelerator for optical neural networks," Nature, vol. 589, no. 7840, pp. 44-51, 2021.

[64] Kues, M. et al. Quantum optical microcombs. Nature Photonics 13, (3) 170-179 (2019). doi:10.1038/s41566-019-0363-0

[65] C. Reimer et al., "Integrated frequency comb source of heralded single photons," Optics Express, vol. 22, no. 6, pp. 6535-6546, 2014

[66] C. Reimer, et al., "Cross-polarized photon-pair generation and bichromatically pumped optical parametric oscillation on a chip", Nature Communications, vol. 6, Article 8236, 2015. DOI: 10.1038/ncomms9236.

[67] L. Caspani, C. Reimer, M. Kues, et al., "Multifrequency sources of quantum correlated photon pairs on-chip: a path toward integrated Quantum Frequency Combs," Nanophotonics, vol. 5, no. 2, pp. 351-362, 2016.

[68] C. Reimer et al., "Generation of multiphoton entangled quantum states by means of integrated frequency combs," Science, vol. 351, no. 6278, pp. 1176-1180, 2016.

[69] M. Kues, et al., "On-chip generation of high-dimensional entangled quantum states and their coherent control", Nature, vol. 546, no. 7660, pp. 622-626, 2017.

[70] P. Roztocki et al., "Practical system for the generation of pulsed quantum frequency combs," Optics Express, vol. 25, no.16, 18940-18949, 2017.

[71] Y. Zhang, et al., "Induced photon correlations through superposition of two four-wave mixing processes in integrated cavities", Laser and Photonics Reviews, vol. 14, no. 7, pp. 2000128, 2020. DOI: 10.1002/lpor.2020001281

[72] C. Reimer, et al.,"High-dimensional one-way quantum processing implemented on d-level cluster states", Nature Physics, vol. 15 (2) 148 (2019).

[73] P. Roztocki et al., "Arbitrary phase access for stable fiber interferometers", Laser and Photonics Reviews, vol.15 (4) 2000524 (2021). DOI: 10.1002/lpor.202000524.

[74] S. Sciara et al., "Generation and Processing of Complex Photon States With Quantum Frequency Combs", IEEE Photonics Technology Letters, vol. 31 (23), 1862-1865 (2019).

[75] P. Roztocki et al., "Complex quantum state generation and coherent control based on integrated frequency combs", Journal of Lightwave Technology, vol. 37 (2), 338-344 (2019).

[76] S Sciara et al., "Universal N-Partite d-Level Pure-State Entanglement Witness Based on Realistic Measurement Settings", Physical Review Letters, Vol. 122 (12), 120501 (2019).

[77] L.Jin, L. Di Lauro, A.Pasquazi, M. Peccianti, David J. Moss, R.Morandotti, B.E. Little, S.T.Chu, "Optical multi-stability in a nonlinear high-order microring resonator filter", Editors Pick, Applied Physics
Letters (APL) Photonics, vol. $\underline{5}$ Article 056106, May 22 (2020). https://doi.org/10.1063/5.0002941

[78] A. Aadhi, Anton V. Kovalev, Michael Kues, Piotr Roztocki, Christian Reimer, Young Zhang, Tao Wang, Brent E. Little, Sai T. Chu, Zhiming Wang, David J. Moss, Evgeny A. Viktorov, and Roberto Morandotti, "Highly reconfigurable hybrid laser based on an integrated nonlinear waveguide", Optics Express, vol. 27 (18) 25252 (2019). DOI: 10.1364/OE.27.025251. 